Annuaire suisse de politique de développement

25-2 | 2006

Paix et sécurité : les défis lancés à la coopération internationale

\title{
Bosnie-Herzégovine. À la recherche d'une vision commune pour un Etat stable
}

\section{René Holenstein}

\section{(2) OpenEdition}

\section{Journals}

Édition électronique

URL : http://journals.openedition.org/aspd/279

DOI : $10.4000 /$ aspd. 279

ISSN : 1663-9669

Éditeur

Institut de hautes études internationales et du développement

Édition imprimée

Date de publication : 1 octobre 2006

Pagination : 223-226

ISBN : 2-88247-064-9

ISSN : $1660-5934$

Référence électronique

René Holenstein, « Bosnie-Herzégovine. À la recherche d'une vision commune pour un Etat stable », Annuaire suisse de politique de développement [En ligne], 25-2 | 2006, mis en ligne le 26 janvier 2010, consulté le 08 septembre 2020. URL : http://journals.openedition.org/aspd/279 ; DOI : https://doi.org/ $10.4000 /$ aspd. 279 


\title{
Bosnie-Herzégovine
}

\author{
A la recherche d'une vision commune pour un Etat stable
}

\section{René Holenstein*}

P lus de dix ans après la fin de la guerre (1992-1995), la Bosnie-Herzégovine souffre de la constitution négociée en 1995 lors des accords de paix de Dayton. Cette constitution divise l'Etat en deux entités politiques relativement autonomes. L'Etat central qui les chapeaute n'est doté que de compétences réduites, ce qui complique l'application rapide des réformes. $60 \%$ des moyens publics sont consacrés à l'administration et ses nombreuses structures parallèles. Les experts sont unanimes: aucun Etat stable ne peut sortir de la constitution actuelle, élaborée dans la précipitation afin de mettre fin à la guerre. L'existence du Bureau du haut représentant (OHR) de la «communauté internationale», organe de surveillance du respect du traité de paix, ravale la BosnieHerzégovine au rang de protectorat. Sa mise sous tutelle politique partielle par l'OHR a empêché les forces locales d'exercer leurs responsabilités politiques, sociales et économiques pour reconstruire l'Etat et la société. Les droits individuels sont mal ancrés dans la constitution; le principe ethnique domine les mécanismes politiques de décision.

\section{Faire participer les citoyens}

Existe-t-il une autre voie? Cette question est au centre du projet «Plate-forme Bosnie-Herzégovine» lancée en avril 2005 par la Direction du développement et de la coopération (DDC) en collaboration avec l'ambassade suisse de Sarajevo. Des séances de discussions publiques ont été organisées à Mostar, à Banja Luka, à Tuzla et dans d'autres villes bosniaques avec le concours d'un journal local de Banja Luka. Les citoyens ont pu venir y prendre position sur les modifications possibles de la constitution. Eu égard à sa propre histoire, la Suisse peut apporter une précieuse contribution à certaines des questions abordées telles que la démocratie directe, le fédéralisme et l'intégration économique et sociale de diverses régions et minorités. Ce projet part du principe que la Bosnie a besoin de pouvoir se représenter son avenir, un avenir liant Croates, Serbes et musulmans. Pour qu'un Etat soit stable, il lui faut une vision commune.

Les discussions ont donné lieu à plusieurs propositions très concrètes sur la forme du futur Etat de Bosnie-Herzégovine; toutes pointent vers la nécessité du changement. Pour de nombreux participants, il est clair que l'unité économique de la Bosnie-Herzégovine est un important préalable à l'intégration politique du pays à l'Europe. Le projet a suscité l'intérêt du public parce que ses principaux artisans sont Bosniaques et non étrangers. Ce qui semble confirmer que, pour être durable, le changement ne doit pas être dirigé d'en haut mais porté et animé

* Chef de la section Gouvernance, Direction du développement et de la coopération (DDC), Berne. 
par la base. Un fait qui a été souligné par des experts suisses tels que Georg Kreis et l'écrivain Adolf Muschg, qui ont pris part aux discussions.

Avec son projet «Plate-forme Bosnie-Herzégovine», la Suisse cherche à insuffler une culture du dialogue dans la discussion sur la constitution. Elle contribue ainsi indirectement à la formation de la nation, laquelle, dans ce pays, n'est pas encore parachevée. Cette approche se fonde sur sa propre expérience de consolidation nationale: au XIX ${ }^{\mathrm{e}}$ siècle, les minorités francophones et italophones ont compris que, intégrées à un Etat suisse unifié, elles seraient en meilleure position que comme simples annexes de la France ou de l'Italie. Le projet «Plateforme Bosnie-Herzégovine» complète judicieusement l'engagement de la Suisse dans le domaine économique et social. De par sa proximité géographique avec la Suisse et en raison de son rapprochement de l'Union européenne (UE), l'Europe du Sud-Est est devenue l'un des grands axes de la politique extérieure suisse. La Suisse s'y est beaucoup engagée, par solidarité avec la population, mais aussi afin de contribuer à la stabilisation de la région des Balkans sur les plans politique, social et économique. La Direction du développement et de la coopération (DDC) et le Secrétariat à l'économie (seco) ont investi ces dernières années quelque 230 millions d'euros dans la région. Au lendemain de la guerre, la priorité est allée à l'aide humanitaire et à la reconstruction des infrastructures. La remise en état des conduites d'eau et d'électricité et la construction d'écoles et d'hôpitaux ont facilité l'intégration des gens chassés par la guerre puis revenus au pays et ont amélioré le sort des populations locales. Entre 1996 et 1999, plus de 15'000 Bosniaques réfugiés en Suisse sont rentrés dans leur patrie. A la fin des années 1990, l'aide d'urgence a été disjointe des programmes de longue durée visant à renforcer la démocratie et l'économie de marché.

La coopération suisse avec la Bosnie-Herzégovine a défini trois domaines d'activité prioritaires pour les années 2004-2008: l'encouragement au développement économique, avant tout des petites et moyennes entreprises, le renforcement des institutions locales sur le plan communal, et le soutien aux réformes dans les domaines de la santé et du social. Actuellement, la DDC et le seco financent deux douzaines d'actions dans ces domaines. Les projets dans les domaines de l'approvisionnement en eau, de la santé et du développement communal ainsi que dans la coopération avec les organisations non gouvernementales améliorent très concrètement la situation de la population. Ils sont ainsi conçus que leur conduite nécessite et favorise la collaboration entre les deux entités républicaines. Concrètement, cela signifie que les ministères des deux entités, ainsi que, selon les projets, les gouvernements cantonaux, les communes et différentes associations y sont représentés. La collaboration de la Suisse avec la Bosnie-Herzégovine participe donc au renforcement de l'ensemble de la nation bosniaque. Les avantages comparatifs de la collaboration suisse sont: un engagement à long terme, un solide savoir-faire en développement et transition, le contact avec la base, le pragmatisme, une participation active entre partenaires et la volonté d'aboutir à des résultats concrets. Ces projets profitent d'autre part de l'expérience suisse en matière de vie communale et de cohabitation entre minorités.

Avec son programme de soutien à la transition en Bosnie-Herzégovine, la Suisse appuie les objectifs du gouvernement bosniaque. La Bosnie-Herzégovine a lancé avec sa stratégie nationale de lutte contre la pauvreté (2004-2007) un 
programme à moyen terme inscrit dans une perspective d'intégration à l'UE. Ce programme de développement est centré sur la réforme fiscale, la croissance du secteur privé, la réduction de l'économie informelle, la protection sociale et la réforme de l'enseignement. Quant aux thèmes transversaux, ils recoupent les mesures contre la corruption, la défense des droits humains et l'égalité du développement des hommes et des femmes.

L'engagement de la Suisse a oscillé ces dernières années autour des 20 millions de francs par an. Son programme de coopération a été soumis en 2005 à l'évaluation du Comité d'aide au développement (CAD) de l'OCDE, qui l'a jugé très efficace. La contribution de la DDC à la révision de la constitution a, en particulier, reçu des louanges. Selon le CAD, il existe un potentiel d'amélioration dans la focalisation thématique qui pourrait être élargie et dans le renforcement des liens entre le travail sur le projet et le programme d'une part, le dialogue politique et la coordination des autres communautés d'autre part.

\section{Enseignements et expériences}

Le développement de la Bosnie-Herzégovine au cours des dernières années montre que la mise du pays sous la haute surveillance d'organisations internationales n'a pas permis d'instaurer des rapports démocratiques stables et durables. Au lendemain de la guerre, les partis politiques qui avaient participé au conflit et dont certains présentaient un caractère mafieux sont restés au pouvoir. Ils ont empêché la réconciliation et tout véritable nouveau commencement. Les forces politiques modérées ne jouent toujours qu'un rôle marginal dans la vie politique. Le parallélisme entre structures internationales et structures nationales a provoqué un syndrome croissant de dépendance. Ainsi, face aux décisions impopulaires, les gouvernements locaux temporisent ou se défaussent sur les autorités internationales. Ce qui ne fait qu'accentuer la passivité et l'indifférence de larges secteurs de la population. Les travailleurs qualifiés restés au pays préfèrent travailler pour l'une des nombreuses organisations internationales dont les salaires sont bien supérieurs à la moyenne locale. Cela vide les institutions étatiques de toute substance; elles ne disposent ni des collaborateurs qualifiés ni des institutions nécessaires pour engager de leur propre chef le processus d'intégration à l'Europe. Elles restent donc extrêmement dépendantes des experts étrangers.

Les sociétés en pleine transformation ont tout particulièrement besoin de conditions favorables au processus de formation démocratique de l'opinion afin de rétablir la confiance, les réseaux sociaux et les réseaux de communication détruits par la guerre. Il faudrait pour cela un concept politique européen assurant l'égalité de tous les citoyens devant la loi et protégeant efficacement les différents groupes de toute discrimination. En matière de formation de l'Etat par la base, la Suisse peut encore renforcer son action dans les pays de l'ancienne Yougoslavie. Le fait qu'il est possible d'intéresser les citoyens à cette question est bien illustré par l'excellent accueil réservé par la population locale au projet «Plate-forme Bosnie-Herzégovine».

La principale découverte de ces quarante années de coopération au développement est sans doute que, sans une vision positive de l'avenir, il n'y a pas de 
développement. C'est pourquoi l'intégration directe de la population à la planification et à la conduite des projets de développement est cruciale. Une véritable participation inclut l'accès égal aux ressources, aux chances, à l'information, au savoir, aux processus de décision et au pouvoir. Et, bien entendu, l'égalité des sexes.

Par ailleurs, la connaissance du contexte local est l'un des facteurs cruciaux pour l'établissement de liens de confiance à long terme. Le simple savoir des experts étrangers ne suffit pas. L'un des facteurs décisifs de succès est le transfert de l'expertise dans le tissu local. L'acceptation et la confiance entre partenaires dans la coopération ne peuvent découler que d'une longue collaboration et ne jaillissent pas de la simple gestion éclair des crises. De nombreux projets et programmes sont trop brefs, trop spécifiques, trop sectoriels. Il faut à l'avenir se montrer encore plus systématique dans l'ouverture des démarches sectorielles et dans l'intégration méthodique des programmes aux structures administratives locales.

Rétrospectivement, l'aide suisse en Bosnie-Herzégovine a bien réussi le passage de l'aide humanitaire à l'aide à la transition. Ainsi, grâce aux fonds de l'Office fédéral des migrations, il a été possible de transformer les projets de retour au pays de personnes déplacées et de réfugiés, anciennement financés par l'aide humanitaire, en collaboration au développement agricole à long terme. Des milliers de places de travail ont pu être créées pour d'anciennes personnes déplacées ou réfugiées.

La Suisse harmonise son travail de coopération avec la stratégie nationale de développement de la Bosnie-Herzégovine. Ce faisant, elle veille en particulier à ce que la société civile participe à l'élaboration et à l'application des programmes. Ainsi, le travail de développement a conduit à des résultats visibles et, pour la population démunie et marginalisée, à des améliorations sensibles, par exemple pour les personnes de retour au pays, les personnes déplacées, le troisième et le quatrième âge ainsi que les enfants défavorisés. 